\title{
Análise da dinâmica inflacionária no Brasil e preços de commodities: uma aplicação do modelo de vetores autorregressivos
}

\author{
Aline Fernanda Soares* \\ Haroldo José Torres da Silva** \\ André Luís Ramos Sanches*** \\ Vitor Augusto Ozaki*****
}

\begin{abstract}
Resumo
O objetivo deste trabalho é investigar a dinâmica inflacionária no Brasil, com ênfase no comportamento dos preços domésticos do grupo alimentação e bebidas dos índices de preços ao consumidor, e sua relação com o comportamento dos preços internacionais de commodities, das taxas de câmbio e de juros e a atividade econômica. Para isto, utiliza-se a metodologia de vetores autorregressivos, com base no período de 2003 e 2013. Os resultados apontam que os preços internacionais de commodities ICAGR e a atividade econômica PIB têm efeito positivo sobre o preço do grupo alimentação e bebidas do índice de preços ao consumidor IPCAF com um período de defasagem.
\end{abstract}

Palavras-chave: Índice de preços. Commodities. Vetores autorregressivos.

* Economista. Mestranda em Economia Aplicada na Escola Superior de Agricultura Luiz de Queiroz, Universidade de São Paulo.E-mail: aline.fernanda.soares@usp.br

** Economista. Mestrando em Economia Aplicada na Escola Superior de Agricultura Luiz de Queiroz, Universidade de São Paulo.E-mail: haroldo.jose.silva@usp.br

**** Economista. Doutorando em Economia Aplicada na Escola Superior de Agricultura Luiz de Queiroz, Universidade de São Paulo. E-mail: andresanches@usp.br

***** Professor do Departamento de Economia, Administração e Sociologia da Escola Superior de Agricultura Luiz de Queiroz, Universidade de São Paulo. E-mail: vitorozaki@usp.br

http://dx.doi.org/10.5335/rtee.v22i46.6758

Submissão: 18/08/2015. Aceite: 21/03/2016. 


\section{Introdução}

A taxa de inflação é um indicador macroeconômico que tem o objetivo de refletir os efeitos sobre a economia do aumento de preços de seus mais variados componentes. Nesse sentido, inflação é uma situação de aumentos contínuos e generalizados dos preços dos bens e serviços em uma economia, de acordo com Bacha (2004) apud Luque e Vasconcellos (1998).

Um índice de preços, segundo Samuelson e Nordhaus (2005), é um indicador de nível médio de preços e a taxa de inflação é a variação do nível geral de preços. Basicamente, os índices de inflação são elaborados a partir da agregação de cestas de variados bens e serviços, existindo assim índices para as diferentes classes de consumidores e diferentes setores da economia.

A existência de um índice geral, conforme aponta Bialoskorski Neto e Ohira (2001), pode distorcer o comportamento dos preços em setores específicos da economia, uma vez que é possível que haja uma aproximação para os setores de uma economia. Marques (1991) argumenta que o sistema de preços existente em uma economia forma um sistema econômico complexo que inter-relaciona variáveis reais, que, por sua vez, geram suas contrapartidas monetárias. Esses movimentos monetários intersetoriais citados pelos autores podem ser medidos pelos índices de preços, que são a comparação entre os preços de diferentes produtos.

Além da relação intersetorial dos preços, é possível destacar a relação dos preços sob a ótica de diferentes mercados e países. Ferrari Filho e Modenesi (2011) atribuem o comportamento da dinâmica inflacionária brasileira em determinados períodos ao aumento nos preços das commodities no mercado internacional, considerada como inflação importada do exterior.

Com a ocorrência de choques negativos de oferta, atrelados à alta expressiva e generalizada dos preços internacionais das commodities, é preciso avaliar as implicações dessa mudança para o comportamento da inflação doméstica, mais especificamente no que tange ao impacto inflacionário de choques nos preços internacionais de commodities.

Além de impulsionar o resultado da balança comercial, é de se esperar que o crescimento das commodities na pauta exportadora tenha ampliado a relevância dos preços internacionais desses itens para o fluxo cambial brasileiro e, por conseguinte, elevado a influência dos preços das commodities sobre a taxa de câmbio e sobre os preços domésticos.

Os consumidores nacionais, em particular a fração mais pobre da população, comprometem uma parcela maior do seu orçamento com alimentos. Nesse sentido, 
trata-se de extrema importância conhecer o processo associado aos aumentos dos preços desse grupo, no caso, alimentação.

Conhecer a dinâmica subjacente à inflação é um passo necessário na avaliação de seus determinantes e dos efeitos de diferentes políticas econômicas. Este trabalho procura respostas analíticas do processo inflacionário principalmente no âmbito do setor agroindustrial, buscando investigar os impactos da alta dos preços dos alimentos e variações nos preços das commodities sobre a dinâmica da inflação no Brasil no período de 2003 a 2013. Especificamente, propõe-se neste trabalho uma análise da dinâmica inflacionária no Brasil, com ênfase no comportamento dos preços do grupo alimentação e bebidas do Índice de Preços ao Consumidor Amplo (IPCA) por meio da aplicação de um modelo de vetores autorregressivos (VAR).

\section{Revisão de literatura}

Martinez (2011) argumenta que a evolução dos preços domésticos de alimentos e bebidas comercializáveis (grupo de maior peso, cerca de 12\% do IPCA) está associada à cotação internacional de commodities e à taxa de câmbio. A variação dos preços de alimentos e bebidas comercializáveis, segundo o autor, segue os preços internacionais de commodities, embora de forma mais moderada. Esse grupo, de acordo com os testes realizados por Martinez e Cerqueira (2011), também responde à demanda interna e às expectativas de inflação. Freitas et al (2001) alega que a trajetória crescente do índice de preços dos alimentos é resultante da ampliação da renda, associada ao crescimento econômico nos países em desenvolvimento, além do descompasso entre a oferta e a expansão da demanda.

Os gastos com alimentação têm participação importante no orçamento mensal do consumidor. Silva e Fonseca Neto, ao analisar a população por segmentos de renda a partir dos dados da Pesquisa de Orçamentos Familiares de 2003, bem como suas cestas de consumo, concluíram que:

[...] os setores alimentação e bebidas e habitação são os mais representativos para as classes mais pobres da população porque são compostos predominantemente por bens de primeira necessidade. Comparativamente aos pobres, as participações destes setores são menores para as classes média e alta. Isso implica que os pobres são mais sensíveis às inflações destes grupos - principalmente o de alimentação e bebidas (2011, p. 91).

Os autores atribuem ainda o comportamento dos preços dos alimentos ao comportamento do preço do petróleo. O aumento no preço do petróleo estimula a produção e a demanda por biocombustíveis, diminuindo a oferta de alimentos (por 
meio da substituição de culturas ou pelo desvio da produção de alimentos para fabricação de álcool).

Com relação aos preços de commodities energéticas e de alimentos no Brasil, Bini e Denardin (2013) analisaram a relação de integração e de causalidade (no sentido de Granger) entre os preços do etanol e petróleo, com as cotações de commodities agrícolas (cana, milho e soja) no Brasil, no período de 2001 a 2012. Os resultados encontrados pelos autores demonstram que os preços das commodities energéticas exercem apenas impactos marginais sobre as commodities agrícolas e indicam também a existência de cointegração entre as commodities agrícolas e o etanol, diferentemente do petróleo que não apresentou essa relação de longo prazo.

Em relação aos preços internacionais de alimentos, Margarido, Serigati e Perosa (2010) mostraram que ocorre transmissão de variações desses preços sobre o comportamento da taxa de câmbio real no Brasil, ou seja, há um relacionamento inverso, no curto prazo, entre as variações nos preços internacionais de alimentos e a taxa de câmbio real. Não obstante, esses autores também observaram, a partir dos testes de cointegração, que não existe relacionamento de longo prazo entre essas variáveis.

O Brasil, por exemplo, é exportador líquido de produtos agrícolas, e, em função dessa situação, há uma relação inversa entre os preços de commodities e taxa de câmbio. De acordo com Lora, Powell e Tavella (2011), a transmissão dos preços internacionais para os preços domésticos depende da reação da taxa de câmbio. Países com sistema de metas de inflação (inflation targeters) tendem a ter taxas de câmbio flexíveis, que podem apreciar-se, amortecendo o aumento nos preços domésticos.

Margarido e Barros (2000), Freitas et al. (2001) e Barbosa, Margarido e Nogueira Junior (2002) mostram que o Brasil é tomador de preços no mercado mundial de commodities agrícolas. O país é um dos principais players no mercado internacional de commodities, entretanto o seu poder de mercado é reduzido.

Os resultados obtidos por Fernandez (2003) também corroboram o argumento de que o Brasil não tem poder de mercado significativo sobre o preço internacional das commodities presentes na sua pauta de exportação, ou seja, as variações da sua taxa de câmbio real não implicam necessariamente em variações contemporâneas dos preços internacionais desses produtos.

É de se esperar que se os preços internacionais aumentem, esse processo também ocorra no mercado doméstico, dado que, conforme mencionado, o Brasil é tomador de preços no mercado mundial de commodities agrícolas. No entanto, segundo Schwartsman (2012), a elevação dos preços das commodities corresponde a 
um aumento dos preços das exportações brasileiras relativamente às importações, dessa forma a melhora de termos de troca tende a fortalecer a moeda, reduzindo o impacto nos preços. Espera-se que, nesse cenário, a variação nos preços de commodities produza movimentos na taxa de câmbio real de um país na medida em que esta mudança altera os termos de troca desse país.

Em países cuja receita oriunda da exportação de matérias-primas é substancial para o fluxo de divisas, conforme aponta Melo (2010), o impacto inflacionário de um choque nos preços das commodities tende a ser atenuado pela apreciação cambial resultante. De forma geral, os aumentos internos dos preços dos alimentos não é um repasse direto da alta internacional das commodities, pois o câmbio se aprecia quando os preços de commodities sobem. Portanto, o aumento dos preços internacionais das commodities impacta nos preços domésticos, mas a apreciação da taxa de câmbio tende a diminuir o impacto sob os preços domésticos.

Entretanto, de acordo com Bastos e Braga (2010), os preços internacionais se mostraram bastantes significativos para a explicação das variações da inflação doméstica. Adicionalmente, os mesmos autores mostraram que, para uma dada taxa de câmbio, um aumento de dez pontos percentuais (p.p.) dos preços dos insumos importados, em dólares, aumenta a inflação em um p.p.

De acordo com dados do Banco Central do Brasil (BCB) (2004), a deterioração nos termos de troca gera efeito renda negativo, reduzindo a renda real, o que resulta em menor demanda por bens não comercializáveis e, para o restabelecimento do equilíbrio, recuo no preço desses bens, levando, portanto, a uma depreciação da taxa de câmbio real.

As estimativas elaboradas por Braga (2013) apontam que a inflação ao consumidor agregada na década de 2000 foi marcada por duas influências principais: a variação cambial (ocorridas, grosso modo, na primeira metade da década) e a evolução dos preços das commodities (relevante principalmente na segunda metade da década). A autora aponta que a inflação importada foi o principal tipo verificado de inflação na economia brasileira na década de 2000.

Nesse contexto, é de grande relevância identificar os padrões da dinâmica dos preços internalizados de commodities, entender a influência de aspectos explicativos relevantes, além de verificar a similaridade da dinâmica dos preços de commodities com o comportamento dos preços domésticos. 


\section{Metodologia}

\subsection{Procedimentos e dados}

As especificações para os testes e modelos econométricos são baseadas nas séries cuja nomenclatura utilizada para as variáveis estão contidas e detalhadas na Tabela 1. As séries utilizadas neste trabalho foram obtidas no Sistema Gerenciador de Séries Temporais (SGC - v2.1) do Banco Central do Brasil. Todas as séries têm frequência mensal, obedecendo-se ao período de análise deste trabalho, isto é, de janeiro de 2003 a dezembro de 2013 (132 observações).

Tabela 1 - Descrição das variáveis utilizadas na pesquisa

\begin{tabular}{|c|c|}
\hline Variável & Definição \\
\hline$P I B$ & $\begin{array}{l}\text { Índice de atividade econômica do Banco Central (IBC-Br) dessazonalizado } \\
\text { (dez./2002 = 100) }\end{array}$ \\
\hline IPCAF & $\begin{array}{l}\text { Índice Nacional de Preços ao Consumidor Amplo (IPCA) dessazonalizado - grupo } \\
\text { alimentação e bebidas (jan./2003 = 100) }\end{array}$ \\
\hline SELIC & Índice da taxa de juros - Selic acumulada no mês (base = jan./2003) \\
\hline CAMBIO & Índice da taxa de câmbio efetiva real (IPA-DI - jun./1994 = 100) \\
\hline ICAGR & Índice de Commodities Brasil Agropecuária (dez./2005 = 100) \\
\hline
\end{tabular}

Fonte: elaborado pelos autores com base nos dados do Banco Central do Brasil (2004).

O IBC-Br é um indicador mensal que procura, com as devidas adaptações, reproduzir o movimento do produto interno bruto (PIB), e, dessa forma, avaliar de forma mais ampla o ambiente macroeconômico do país. Neste trabalho, o IBC-Br é utilizado como uma proxy para o PIB.

O Índice de Commodities Brasil (IC-Br $)^{1}$ é elaborado pelo Banco Central do Brasil, a partir dos preços das commodities convertidos em reais, de modo a identificar a parcela das variações de preços das commodities no mercado externo relevante para explicar a dinâmica da inflação doméstica.

O IC-Br exprime a média mensal ponderada dos preços em reais das principais commodities relevantes para a dinâmica da inflação brasileira, é segmentado em três subíndices (IC-Br Agropecuária, IC-Br Metal e IC-Br Energia) e abrange somente 19 commodities exportadas pelo Brasil. Neste trabalho restringiu-se à utilização do IC-Br Agropecuária, isto é, um subíndice do IC-Br elaborado a partir da média mensal ponderada dos preços em reais da carne de boi, algodão, óleo de soja, trigo, açúcar, milho, café, arroz e carne de porco. 


\subsection{Procedimentos econométricos}

O objetivo desta seção é definir o instrumental metodológico que foi adotado: testes de raiz unitária, de cointegração, de causalidade de Granger, e o modelo VAR. Utilizou-se o software Eviews versão 8.0 e o software R versão 3.0.1 para a obtenção dos resultados econométricos.

\subsection{O modelo de vetores autorregressivos}

O modelo de série temporal multivariado ${ }^{2}$ para as variáveis macroeconômicas utilizado neste artigo é um modelo de vetores autorregressivos (vector auto-regressive models), baseado somente na componente autorregressiva, isto é, $X_{t}$ é descrito apenas por seus valores passados e pelo vetor de ruído branco.

Pode-se expressar um modelo autoregressivo de ordem $p$ por um vetor com $n$ variáveis endógenas, $X_{t}$, conectadas entre si por meio de uma matriz $B$, conforme a Equação (1).

$$
B X_{t}=\Gamma_{0}+\sum_{i=1}^{p} \Gamma_{i} X_{t-i}+\varepsilon_{t}
$$

Em que:

$B$ é uma matriz $n \times n$ que define as restrições contemporâneas entre as variáveis que constituem o vetor $n \times 1, B X_{t}$;

$\Gamma_{0}$ é um vetor de constantes $n \times 1$;

$\Gamma_{\mathrm{i}}$ são matrizes dos coeficientes das variáveis defasadas $\mathrm{n} \times \mathrm{n}$;

$\varepsilon_{t}$ é um vetor $n \times 1$ de perturbações aleatórias não correlacionadas entre si contemporânea ou temporalmente.

As hipóteses acerca de $\varepsilon_{t}$ são: média zero, variância constante $(\varepsilon \approx N(0, \sigma)$ e ausência de autocorrelação $\left[\operatorname{Cov}\left(\varepsilon_{t}, \varepsilon_{j}\right)=0\right]$.

De acordo com Enders (1996), a equação (1) expressa as relações entre as variáveis endógenas, a partir de um modelo econômico teoricamente estruturado. Multiplicando-se toda a equação (1) por $B^{-1}$, tem-se o modelo estimado em sua forma reduzida tal como expresso pela equação (2):

$$
X_{t}=\mathrm{A}_{0}+\sum_{i=1}^{p} A_{i} X_{t-i}+e_{t}
$$


Em que:

$$
\begin{aligned}
& A_{0}=B^{-1} \Gamma_{0} ; \\
& A_{i}=B^{-1} \Gamma_{i} ; \\
& e_{t}=B^{-1} \varepsilon_{t} ;
\end{aligned}
$$

Um VAR ( $p$ ), por exemplo, com $n$ variáveis endógenas teria $n+n^{2} p$ coeficientes a serem estimados, uma vez que as matrizes $A_{i}$ têm dimensão $n \times n$ e as $n$ primeiras variáveis referem-se à constante, sem contar ainda os coeficientes de possíveis variáveis exógenas. Conforme elucidado por Enders (1996), uma vez que o lado direito do sistema (8) contém apenas variáveis predeterminadas e os termos de erros são assumidos serialmente não correlacionados e com variância constante, pode-se estimar cada equação desse sistema por OLS (Ordinary Least Squares).

\subsection{Teste de Granger - causalidade}

A metodologia utilizada baseia-se no procedimento proposto por Toda e Yamamoto (1995) para testar a hipótese de não causalidade de Granger. É importante mencionar que não se propõe identificar uma relação de causalidade no seu sentido de endogeneidade por meio do teste de causalidade de Granger - veja Maddala (1992). Neste trabalho, as cinco variáveis macroeconômicas foram definidas, $a$ priori, como endógenas na formulação dos modelos, tal como o VAR. ${ }^{3}$

\section{Resultados}

A partir de 2010, a alta de preços das commodities intensifica-se ao mesmo tempo em que a política de acumulação de reservas gera impacto na apreciação do câmbio. Esses dois fatores, aliados ao crescimento do PIB, geraram cenário favorável ao aumento dos preços internos de alimentos, como mostra a curva do índice de commodities IPCAF. Segundo Martinez (2011), a valorização do câmbio real reflete em parte os ganhos no valor das exportações com o aumento dos preços de commodities. Na Figura 1, verifica-se a evolução das variáveis PIB, IPCAF, SELIC, CAMBIO e ICAGR entre 2003 e 2013.

Com a alta de preços das commodities e apreciação do câmbio, os preços dos alimentos no mercado interno aumentaram no período analisado, como mostra a curva do índice de commodities IPCAF na Figura 1. 
Figura 1 - Evolução temporal, de janeiro de 2003 a dezembro de 2013, das variáveis macroeconômicas utilizadas no modelo (PIB, IPCAF, SELIC, CAMBIO e ICAGR)

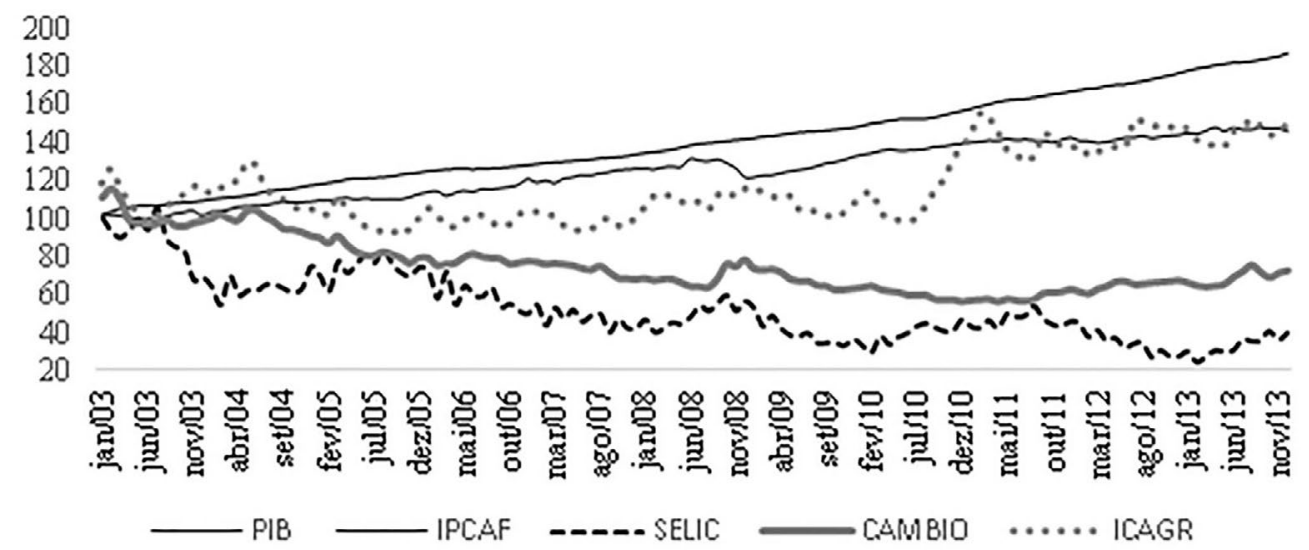

Fonte: elaborado pelos autores a partir de BANCO CENTRAL DO BRASIL (2004).

Inicialmente, analisou-se a sazonalidade das variáveis utilizadas no trabalho, os resultados do teste são apresentados na Tabela 2. No caso da existência de raízes unitárias sazonais, conforme demonstrado por Engle, Granger e Hallman (1989), o método de Engle e Granger (1987) torna-se inapropriado para testar a cointegração, pois os resultados podem ser viesados.

Tabela 2 - Resultados do teste de sazonalidade para as séries PIB, IPCAF, SELIC, CAMBIO e ICAGR, janeiro de 2003 a dezembro de 2013

\begin{tabular}{|c|c|c|c|c|c|}
\hline \multirow[t]{2}{*}{ Série } & \multirow{2}{*}{\begin{tabular}{|c} 
Teste F sazonalidade \\
estável
\end{tabular}} & \multicolumn{2}{|c|}{$\begin{array}{l}\text { Teste sazonalidade } \\
\text { estável Kruskal Wallis }\end{array}$} & \multirow{2}{*}{\begin{tabular}{|c|}
$\begin{array}{c}\text { Teste sazonalidade } \\
\text { móvel }\end{array}$ \\
F \\
\end{tabular}} & \multirow{2}{*}{$\begin{array}{l}\text { Teste combinado sazonalidade } \\
\text { identificável }\end{array}$} \\
\hline & & T1 & Prob. & & \\
\hline IPCAF & 2,331 & 29,5448 & 0,002 & 2,803 & Não identificável \\
\hline PIB & 0,870 & 7,3615 & 0,769 & 3,472 & Não identificável \\
\hline SELIC & 13,666 & 65,3409 & 0,000 & 0,577 & Identificável \\
\hline CAMBIO & 0,644 & 10,1299 & 0,519 & 2,748 & Não identificável \\
\hline ICAGR & 6,322 & 52,2692 & 0,000 & 1,393 & Provavelmente não identificável \\
\hline
\end{tabular}

Fonte: elaborada pelos autores com resultados da pesquisa.

Conclui-se que apenas a taxa de juros é passível de ajuste sazonal, o que justifica o uso da série dessazonalizada nas etapas seguintes do estudo. Por se tratar de dados econômicos, cuja variância pode ser heterocedástica, aplica-se logaritmo neperiano nas séries a fim de estabilizar a variância. 
Os testes de raiz unitária Dickey-Fuller Aumentado (ADF), Phillips-Perron, Dickey-Fuller GLS e NG-Perron foram realizados a fim de verificar a estacionariedade das séries. Os resultados dos três últimos testes podem ser encontrados no Anexo A, enquanto o resultado do primeiro é discutido a seguir.

O número de defasagens $(p)$ empregado em cada teste de raiz unitária foi determinado pelo critério de Schwarz (SIC). Os resultados, apresentados na Tabela 3 , indicam que não se deve rejeitar a hipótese nula ao considerar o logaritmo neperiano das séries, ou seja, elas são não estacionárias em nível. Para a primeira diferença de todas as séries, rejeita-se a existência de raiz unitária. Os outros testes implementados com o objetivo de analisar a estacionariedade das séries, apresentados no Anexo A, corroboram os resultados encontrados no teste ADF.

Os testes de raiz unitária considerados no trabalho indicam que as séries podem ser consideradas integradas de ordem um I(1), em função da necessidade da aplicação de uma diferença para torná-las estacionárias, possibilitando assim a realização do teste de cointegração de Johansen nas variáveis do modelo.

Tabela 3 - Resultados do teste de raiz unitária Dickey-Fuller Aumentado (ADF) para as séries PIB, IPCAF, SELIC, CAMBIO e ICAGR, janeiro de 2003 a dezembro de 2013

\begin{tabular}{|c|c|c|c|c|c|c|c|c|}
\hline \multirow{2}{*}{ Série } & \multicolumn{3}{|c|}{$\begin{array}{l}\text { Modelo com constate } \\
\text { e tendência }\end{array}$} & \multicolumn{3}{|c|}{$\begin{array}{l}\text { Modelo com } \\
\text { constante }\end{array}$} & \multirow{2}{*}{$\begin{array}{c}\begin{array}{c}\text { Passeio } \\
\text { aleatório }\end{array} \\
\tau\end{array}$} & \multirow{2}{*}{$p$} \\
\hline & $\tau_{\beta}$ & $\theta_{3}$ & $\tau_{\beta}$ & $\tau_{\mu}$ & $\theta_{1}$ & $\tau_{\alpha}$ & & \\
\hline \multicolumn{9}{|l|}{ Nível } \\
\hline PIB & $-2,10$ & 2,44 & 1,94 & $-1,05$ & 6,22 ** & 1,13 & 3,34 & 0 \\
\hline IPCAF & $-1,75$ & 4,45 & 2,20 ** & 1,99 & $12,83^{* * *}$ & $-1,72$ & 4,73 & 2 \\
\hline SELIC & $-3,44$ & 6,25 & $-2,71$ *** & $-2,21$ & 2,68 & $2,15^{* *}$ & $-0,85$ & 3 \\
\hline CÂMBIO & $-1,05$ & 2,84 & 0,24 & $-2,38$ & 3,71 & $2,32 * *$ & $-1,40$ & 0 \\
\hline ICAGR & $-3,38$ & 6,40 & $3,13^{* * *}$ & $-1,67$ & 1,42 & 1,67 & 0,20 & 1 \\
\hline \multicolumn{9}{|c|}{ Diferença } \\
\hline $\mathrm{PIB}^{1}$ & $-11,66$ *** & $67,99 * * *$ & $-0,71$ & $-11,66^{* * *}$ & 68,02 *** & 3,31 *** & $-6,29 * * *$ & 0 \\
\hline $\mathrm{IPCAF}^{2}$ & $-7,23^{* \star *}$ & 26,19 *** & 2,40 ** & $-6,70^{* * *}$ & $22,47^{* * *}$ & $4,61^{* \star *}$ & $-4,13^{* * *}$ & 1 \\
\hline SELIC & $-3,97^{* *}$ & $7,88^{* *}$ & 0,77 & $-3,90 * * *$ & 7,63 *** & $-0,67$ & $-3,86 * * *$ & 2 \\
\hline CÂMBIO & $-10,02^{* * *}$ & 50,22 *** & 2,13 ** & $-9,66$ *** & $46,67^{* * *}$ & $-1,22$ & $-9,57^{* * *}$ & 0 \\
\hline ICAGR & $-8,50^{* * *}$ & $36,17^{* * *}$ & 1,12 & $-8,42^{* * *}$ & 35,48 *** & 0,25 & $-8,45^{* * *}$ & 0 \\
\hline
\end{tabular}

Fonte: elaborada pelos autores com resultados da pesquisa.

Nota: ${ }^{* * *}$ significativo a $1 \% ;{ }^{* *}$ significativo a $5 \% ;{ }^{1}$ lag do passeio aleatório foi $1 ;{ }^{2}$ lag do passeio aleatório foi 0. 
A ordem de defasagens inserida no modelo de cointegração de Johansen foi baseada na análise dos menores valores dos critérios de informação de Schwarz (SC), de Hannan e Quinn (HQ) e da correlação dos resíduos. Para isto, estimou-se um modelo VAR irrestrito, com 2 lags, a partir das séries PIB, IPCAF, SELIC, CÂMBIO e ICAGR, em nível. Embora os critérios de SC e de HQ tenham sugerido uma defasagem, o teste multiplicador de Lagrange de correlação dos resíduos indicou quatro defasagens. Dessa forma, optou-se pela utilização de quatro defasagens para realizar os testes de Johansen e de causalidade de Granger bem como estimar o modelo de vetores autorregressivos.

O teste de Johansen, por meio da estatística do traço e do máximo autovalor, indicou que não existe nenhum vetor de cointegração $(r=0)$ entre as séries analisadas (Tabela 4). O teste do traço constatou que, ao nível de significância de 5\%, não se pode rejeitar a hipótese nula de que não há nenhum vetor de cointegração.

Tabela 4 - Resultados do teste de cointegração de Johansen entre as séries PIB, IPCAF, SELIC, CAMBIO e ICAGR, janeiro de 2003 a dezembro de 2013

\begin{tabular}{l|c|c|c|c|c|c}
\hline \multirow{2}{*}{$\begin{array}{c}H_{0}: \text { r vetores } \\
\text { de cointegração }\end{array}$} & \multicolumn{3}{|c|}{ Teste do traço } & \multicolumn{2}{c}{ Teste do máximo autovalor } \\
\cline { 2 - 6 } & \multicolumn{3}{|c|}{$\lambda_{\text {traço }}(r)=-T \sum_{i=r+1}^{n} \ln \left(1-\widehat{\left.\lambda_{l}\right)}\right.$} & $\lambda_{\text {max }}(r, r+1)=-T \ln \left(1-\widehat{\lambda_{r+1}}\right)$ \\
\cline { 2 - 6 } & Estatística & $\begin{array}{c}\text { Valor crítico } \\
(5 \%)\end{array}$ & Prob. $^{1}$ & Estatística & $\begin{array}{c}\text { Valor crítico } \\
(5 \%)\end{array}$ & Prob. $^{1}$ \\
\hline$r=0$ & 67,468 & 69,819 & 0,076 & 28,145 & 33,877 & 0,207 \\
$r \leq 1$ & 39,322 & 47,856 & 0,248 & 21,016 & 27,584 & 0,275 \\
\hline
\end{tabular}

Fonte: elaborada pelos autores com resultados da pesquisa.

Nota:1 p-valor baseado em MacKinnon-Haug-Michelis (1999).

Nesse mesmo sentido, o teste do máximo autovalor também revelou que a hipótese nula não deve ser rejeitada. O teste pairwise de causalidade de Granger, cujos resultados para as séries em análise estão apresentados na Tabela 5, indica a precedência temporal entre as variáveis. Ao nível de significância de 5\%, rejeita-se a hipótese nula de que o índice de commodities agrícolas não causa, no sentido de Granger, o índice de inflação para alimentos e bebidas, ou seja, mudanças no ICAGR devem impactar o IPCAF. Do mesmo modo, as hipóteses nulas de que as taxas de câmbio e de juros, ambas convertidas em índice, não causam no sentido 
de Granger o índice de atratividade econômica são rejeitadas. Quanto à relação de causalidade das demais séries, não se pode inferir estatisticamente que há uma relação de causalidade no sentido de Granger entre elas.

Tabela 5 - Resultados do teste pairwise de causalidade de Granger para as séries PIB, IPCAF, SELIC, CAMBIO e ICAGR, janeiro de 2003 a dezembro de 2013

\begin{tabular}{l|c|c|c|c|c|c|c|c|c|c}
\hline \multirow{2}{*}{ Série } & \multicolumn{2}{|c|}{ PIB } & \multicolumn{2}{c|}{ IPCAF } & \multicolumn{2}{c|}{ SELIC } & \multicolumn{2}{c|}{ CAMBIO } & \multicolumn{2}{c}{ ICAGR } \\
\cline { 2 - 10 } & $X^{2}$ & Prob. & $X^{2}$ & Prob. & $X^{2}$ & Prob. & $X^{2}$ & Prob. & $X^{2}$ & Prob. \\
\hline PIB & - & - & 3,622 & 0,460 & 8,635 & 0,071 & 7,951 & 0,093 & 0,932 & 0,920 \\
IPCAF & 5,339 & 0,254 & - & - & 2,349 & 0,672 & 2,489 & 0,647 & 0,846 & 0,932 \\
SELIC & 18,536 & 0,001 & 9,372 & 0,053 & - & - & 2,330 & 0,675 & 0,933 & 0,920 \\
CAMBIO & 16,276 & 0,003 & 2,378 & 0,667 & 0,504 & 0,973 & - & - & 1,171 & 0,883 \\
ICAGR & 8,625 & 0,071 & 10,726 & 0,030 & 4,833 & 0,305 & 5,827 & 0,212 & - & - \\
\hline
\end{tabular}

Fonte: elaborada pelos autores com resultados da pesquisa.

Após analisar a cointegração de Johansen e relação de causalidade de Granger das séries, foi feita a análise multivariada das séries por meio do modelo de vetor autorregressivo. O VAR capta as relações das variáveis endógenas em análise, para isso, faz-se necessário classificar as séries PIB, IPCAF, SELIC, CAMBIO e ICAGR quanto à endogeneidade/exogeneidade para que o modelo seja corretamente especificado. Neste trabalho, todas as séries foram consideradas endógenas conforme procedimento seguido pelo Banco Central do Brasil (2004), bem como obedecendo aos resultados da decomposição de Cholesky para a ordenação das variáveis no VAR.

$\mathrm{Na}$ equação (3) são a apresentados os principais resultados do modelo VAR estimado.

$$
\left[\begin{array}{c}
\text { DPIB } \\
\text { DIPCAF } \\
\text { DSELIC } \\
\text { DCAMBIO } \\
\text { DICAGR }
\end{array}\right]_{t}=\left[\begin{array}{c}
0,003 \\
0,002 \\
-0,027 \\
-0,006 \\
0,005
\end{array}\right]+\left[\begin{array}{ccccc}
-0,136 & 0,180 & -0,096 & -0,027 & 0,003 \\
0,060 & 0,690 & -0,014 & -0,011 & 0,002 \\
1,815 & 1,273 & 0,026 & -0,379 & -0,027 \\
-0,010 & -0,340 & 0,131 & -0,002 & -0,006 \\
0,063 & 0,096 & -0,039 & 0,028 & 0,005
\end{array}\right]\left[\begin{array}{c}
\text { DPIB } \\
\text { DIPCAF } \\
\text { DSELIC } \\
\text { DCAMBIO } \\
\text { DICAGR }
\end{array}\right]_{t-1}+\left[\begin{array}{c}
e_{P I B} \\
e_{I P C A F} \\
e_{S E L I C} \\
e_{C A M B I O} \\
e_{I C A G R}
\end{array}\right]_{t}
$$

Na Tabela 6 é possível observar a estimação do VAR proposto, os valores entre colchetes são as estatísticas $t$. Os valores estimados para o modelo VAR(4) indexado pelo mês t é: 
Tabela 6 - Resultados do modelo VAR para as variáveis DPIB, DIPCAF, DCAMBIO, DICAGR, DSELIC, janeiro de 2003 a dezembro de 2013

\begin{tabular}{|c|c|c|c|c|c|}
\hline \multirow{2}{*}{$\begin{array}{c}\text { Variáveis } \\
\text { independentes }\end{array}$} & \multicolumn{5}{|c|}{ Variáveis dependentes } \\
\hline & DPIB & DIPCAF & DCAMBIO & DICAGR & DSELIC \\
\hline \multirow{2}{*}{ DPIB(-1) } & $-0,135995$ & 0,059639 & $-0,010473$ & 0,063409 & 1,814962 \\
\hline & {$[-1.42923]$} & [1.16536] & {$[-0.03572]$} & [0.15269] & [2.92195] \\
\hline \multirow{2}{*}{ DPIB(-2) } & 0,075352 & 0,027925 & $-0,439869$ & $-0,440111$ & $-0,237262$ \\
\hline & [ 0.79943] & [0.55085] & {$[-1.51447]$} & {$[-1.06986]$} & {$[-0.38560]$} \\
\hline \multirow{2}{*}{ DPIB(-3) } & $-0,051296$ & $-0,002898$ & 0,126610 & $-0,097580$ & 0,444066 \\
\hline & {$[-0.57575]$} & {$[-0.06048]$} & [0.46118] & {$[-0.25095]$} & [0.76352] \\
\hline \multirow{2}{*}{ DPIB(-4) } & $-0,136691$ & 0,053893 & 0,545525 & $-0,078710$ & 0,191911 \\
\hline & {$[-1.56660]$} & [1.14843] & [2.02902] & {$[-0.20669]$} & [0.33693] \\
\hline \multirow{2}{*}{ DIPCAF(-1) } & 0,180268 & 0,689530 & $-0,340216$ & 0,095704 & 1,272690 \\
\hline & [1.02195] & [7.26808] & {$[-0.62592]$} & [0.12431] & [1.10525] \\
\hline \multirow{2}{*}{ DIPCAF(-2) } & $-0,298207$ & $-0,189715$ & 0,158674 & 0,196599 & 0,135258 \\
\hline & {$[-1.41858]$} & {$[-1.67800]$} & [0.24496] & [0.21429] & [0.09857] \\
\hline \multirow{2}{*}{ DIPCAF(-3) } & 0,282097 & $-0,167803$ & 0,278484 & $-0,011686$ & $-0,316739$ \\
\hline & [1.32983] & {$[-1.47079]$} & [0.42604] & {$[-0.01262]$} & {$[-0.22873]$} \\
\hline \multirow{2}{*}{ DIPCAF(-4) } & $-0,261568$ & 0,198947 & 0,577434 & $-0,599218$ & 2,254676 \\
\hline & {$[-1.49780]$} & [ 2.11816] & [1.07306] & {$[-0.78620]$} & [1.97778] \\
\hline \multirow{2}{*}{ DCAMBIO(-1) } & $-0,095917$ & $-0,014023$ & 0,130683 & $-0,039189$ & 0,025747 \\
\hline & {$[-2.78709]$} & {$[-0.75761]$} & [1.23232] & {$[-0.26091]$} & [0.11460] \\
\hline \multirow{2}{*}{ DCAMBIO(-2) } & $-0,023984$ & 0,025683 & $-0,043161$ & $-0,119073$ & 0,137542 \\
\hline & {$[-0.69800]$} & [1.38977] & {$[-0.40765]$} & {$[-0.79403]$} & [0.61320] \\
\hline \multirow{2}{*}{ DCAMBIO(-3) } & $-0,022899$ & $-0,004477$ & $-0,050906$ & $-0,152459$ & $-0,052982$ \\
\hline & {$[-0.66853]$} & {$[-0.24303]$} & {$[-0.48231]$} & {$[-1.01984]$} & {$[-0.23695]$} \\
\hline \multirow{2}{*}{ DCAMBIO(-4) } & $-0,061771$ & 0,018886 & 0,032489 & $-0,021853$ & $-0,008639$ \\
\hline & {$[-1.82932]$} & [1.03993] & {$[0.31225]$} & {$[-0.14829]$} & {$[-0.03919]$} \\
\hline \multirow{2}{*}{ DICAGR(-1) } & 0,051670 & 0,020733 & 0,008144 & 0,321789 & $-0,203288$ \\
\hline & [2.07729] & [1.54975] & [0.10626] & [2.96420] & {$[-1.25197]$} \\
\hline \multirow{2}{*}{ DICAGR(-2) } & $-0,043623$ & 0,001498 & $-0,027830$ & $-0,083344$ & $-0,171029$ \\
\hline & {$[-1.63945]$} & [0.10464] & {$[-0.33943]$} & {$[-0.71769]$} & {$[-0.98465]$} \\
\hline \multirow{2}{*}{ DICAGR(-3) } & 0,020867 & 0,024701 & 0,101235 & 0,122628 & 0,117895 \\
\hline & [0.80753] & [1.77738] & [1.27141] & [ 1.08736$]$ & [0.69892] \\
\hline \multirow{2}{*}{ DICAGR(-4) } & 0,016951 & $-0,003799$ & $-0,178893$ & $-0,207650$ & 0,108702 \\
\hline & [0.67360] & {$[-0.28071]$} & {$[-2.30703]$} & {$[-1.89068]$} & [0.66172] \\
\hline \multirow{2}{*}{ DSELIC(-1) } & $-0,026680$ & $-0,011193$ & $-0,002426$ & 0,028426 & $-0,379471$ \\
\hline & {$[-1.81463]$} & {$[-1.41552]$} & {$[-0.05355]$} & [ 0.44299] & [-3.95370] \\
\hline \multirow{2}{*}{ DSELIC(-2) } & $-0,026283$ & 0,004480 & $-0,020722$ & 0,035796 & 0,137094 \\
\hline & {$[-2.13972]$} & [0.67819] & {$[-0.54748]$} & [0.66771] & [1.70972] \\
\hline \multirow{2}{*}{ DSELIC(-3) } & $-0,032203$ & $-0,001800$ & 0,017262 & 0,012578 & 0,575976 \\
\hline & {$[-2.58502]$} & {$[-0.26872]$} & [0.44967] & [ 0.23134$]$ & [7.08262] \\
\hline \multirow{2}{*}{ DSELIC(-4) } & 0,009965 & 0,004027 & 0,028730 & 0,006572 & 0,100598 \\
\hline & [0.68703] & [0.51615] & [0.64279] & [0.10382] & [1.06243] \\
\hline \multirow{2}{*}{ C } & 0,002964 & 0,002010 & $-0,005997$ & 0,005376 & $-0,026880$ \\
\hline & [2.12339] & [2.67736] & {$[-1.39439]$} & [0.88252] & {$[-2.95002]$} \\
\hline
\end{tabular}

Fonte: elaborada pelos autores com resultados da pesquisa. 
Os testes de diagnóstico dos resíduos do modelo (teste de normalidade, teste de LM de correlação e teste de máximo autovalor de estabilidade do modelo) são apresentados no Anexo A.

Uma vez que as variáveis foram transformadas por logaritmo neperiano, é possível interpretar os resultados como elasticidades. Um aumento de $1 \%$ no PIB e no IPCAF impacta positivamente a SELIC em 1,815\% e 1,273\%, respectivamente. Por outro lado, um aumento de $1 \%$ no CAMBIO e no ICAGR causa uma queda na SELIC de $0,379 \%$ e $0,027 \%$, conforme exposto equação (3).

Os sinais das variáveis estão de acordo com o esperado. O IPCAF e o ICAGR devem impactar positivamente o PIB com um período de defasagem. Por outro lado, SELIC e CÂMBIO devem ter um efeito negativo no PIB. Quando a economia está aquecida, a inflação tende a aumentar e como medida de controle, o governo aumenta a taxa de juros, a qual encarece os empréstimos, portanto, desestimula os investimentos e retrai a economia.

Com relação ao impacto no IPCAF, PIB e ICAGR devem ter efeito positivo e SELIC e CÂMBIO, negativo com um período de defasagem. Isto porque um aquecimento da atividade econômica aumenta a demanda por bens e serviços, pressionando os preços dos alimentos e bebidas. No mesmo sentido, maiores preços das commodities agrícolas influenciam os preços dos alimentos e bebidas. Já a taxa de juros, conforme explicado acima, seu aumento retrai a economia, o que por sua vez, diminui o nível dos preços. O aumento da taxa de câmbio também tem um impacto negativo na inflação com um período de defasagem, já que o Brasil é pouco dependente de produtos importados e o país é um dos maiores exportadores de commodities agrícolas do mundo, dessa forma, a desvalorização da taxa de câmbio deve impactar negativamente a taxa de inflação.

Este trabalho é mais um passo na tentativa de obter mais detalhes quanto à dinâmica de preços no Brasil, os resultados encontrados complementam as estimativas encontradas por Souza, Annegues e Maia (2015), Maluf e Speranza (2013), e Martinez (2011). Souza, Annegues e Maia (2015) relacionaram o comportamento da taxa de câmbio, da taxa de juros, do PIB, e inflação esperada, à dinâmica da inflação brasileira, enfatizando o papel dos preços domésticos de alimentos. Os autores demonstram que choques na política monetária, no PIB e no câmbio tem efeitos no comportamento da inflação medidos pelo IPCA, maior no curto prazo.

Maluf e Speranza (2013), evidenciam a transmissão das variações dos preços internacionais das commodities alimentares para os preços domésticos dos alimentos, caracterizam o processo de transmissão lenta e limitada de imediato, 
mas transmissão plena no médio prazo, no qual o câmbio apresenta fundamental importância.

Martinez (2011) discute a dinâmica inflacionária brasileira entre os anos 2000 e 2011. O autor verifica que os efeitos da alta internacional dos preços de commodities associado ao comportamento do câmbio levou as taxas de variação dos preços domésticos de alimentos e bebidas a níveis acima da meta de inflação, em especial para o Índice Nacional de Preços ao Consumidor Amplo - IPCA.

\section{Considerações finais}

Buscou-se, com este estudo, analisar as implicações da mudança na correlação entre preços das commodities e da taxa de câmbio brasileira sobre a inflação doméstica (em especial, sobre os preços de alimentos e bebidas), ou seja, com o câmbio vinculado aos preços das commodities, procurou-se verificar qual o repasse (pass-through) na inflação brasileira de um choque nos preços das commodities.

Com a estimação de vetores autorregressivos foi possível alcançar esse objetivo. Embora não conste no artigo, é possível explorar pormenorizado esses detalhes com a análise da decomposição da variância e das respostas a impulsos entre as variáveis analisadas.

Dentre os fatores explicativos da dinâmica inflacionária brasileira no período recente, destaca-se a alta dos preços dos alimentos a variação nas cotações das commodities. A alta dos preços de commodities e o fortalecimento do mercado interno geram pressões inflacionárias, dessa forma, as medidas oriundas da política cambial e monetária devem ser condizentes com o atual contexto econômico.

As evidências encontradas neste trabalho apontam para uma possível associação entre preços de commodities e a inflação doméstica: estimou-se um repasse de $2,07 \%$ do índice de commodities em reais, para preços de alimentos no IPCA, isto é, refletindo que não é um repasse integral.

Sugere-se para pesquisas futuras o uso das metodologias apresentadas neste trabalho, com a finalidade de identificar os condicionantes da dinâmica inflacionária, bem como pelo uso da técnica econométrica de VAR. A participação do grupo alimentação e bebidas na cesta de consumo das famílias é expressiva, com destaque para as famílias de baixa renda. Os efeitos deletérios dos aumentos de preços, e a consequente redução do poder de compra da população, estão intimamente associados às questões de bem-estar, portanto devem ser mote de estudos e profunda atenção da autoridade monetária no controle da dinâmica dos preços. 


\title{
Analisys of inflation dynamics in Brazil and pricing commodities: a vectors autoregressive model application
}

\begin{abstract}
The objective of this study is to investigate the inflation dynamics in Brazil, with emphasis on the behavior of domestic prices of the group "food and beverage" of the consumer prices indices, as well as the influence of the exchange rate, interest rate and economic activity. This paper uses empirical methodology of Vector Autoregressive, based on the period from 2003 to 2013. The results show that international prices of commodities ICAGR and economic activity PIB have positive effect on the price of the group food and beverage of the consumer prices index IPCAF with one lag period.
\end{abstract}

Keywords: Price index. Commodities. Vector Auto Regressive.

\section{Análisis de la dinámica de la inflación en Brasil y precios de commodities: una aplicación del modelo de vectores autoregressivos Resumen}

El objetivo de ese trabajo es investigar la dinámica inflacionaria en Brasil, con énfasis en el comportamiento de los precios domésticos del grupo "comida y bebidas" de los índices de precios al consumidor, y la relación de este con el comportamiento de los precios internacionales de commodities, de la tasa de cambio, tasa de interés y la actividad económica. Para esto, se utiliza la metodología de Vectores Autoregresivos, con base en el periodo de 2003 y 2013. Los resultados indican que los precios internacionales de commodities ICAGR y la actividad económica PIB tienen efecto positivo sobre el precio del grupo "comida y bebidas" del índice de precios al consumidor IPCAF con un periodo de retraso.

Palabras-llave: Índice de precios. Commodities. Vectores autoregresivos. 


\section{Notas}

1 Para mais detalhes, inclusive sobre a metodologia de construção do índice, ver o boxe Repasse dos preços das commodities para o IPCA e índice de Commodities Brasil (IC-Br) do Relatório de inflação do Banco Central de dezembro de 2010.

2 Os modelos econômicos, em geral, são expressos por meio de diversas variáveis (BUENO, 2011). Portanto, o uso de modelos univariados é limitado para expressar alguns modelos, tal como o apresentado no âmbito deste trabalho.

3 O Departamento de Estudos e Pesquisas do Banco Central do Brasil desenvolve alguns modelos VAR que, a cada mês, geram previsões para a inflação dos preços livres. Nesses modelos, consideram-se como variáveis exógenas, a saber, a constante, as dummies de tendência e as dummies sazonais (BANCO CENTRAL DO BRASIL, 2004).

\section{Referências}

BANCO CENTRAL DO BRASIL. Relatório de inflação, Brasília, v. 6, n. 2, jun. 2004. Disponível em: <http://www.bcb.gov.br/htms/relinf/port/2004/06/ri200406b8p.pdf>. Acesso em: 17 dez. 2014.

BARBOSA, M. Z.; MARGARIDO, M. A.; NOGUEIRA JUNIOR, Sebastião. Análise da dinâmica da formação de preços no mercado brasileiro de algodão. Nova Economia, Belo Horizonte, v. 12, n. 2, p. 79-108, jul./dez. 2002.

BASTOS, C. P. M.; BRAGA, J. M. Conflito distributivo e inflação no Brasil: uma aplicação ao período recente. In: BASTOS, C. P. M.; BRAGA, J. M. Macroeconomia para o desenvolvimento: crescimento, estabilidade e emprego. Brasília: Instituto de Pesquisa Econômica Aplicada, 2010. p. 119-153. cap. 4.

BIALOSKORSKI NETO, S.; OHIRA, T. H. Importância e metodologia de regionalização de índice de preços ao produtor: o caso da macro-região de Ribeirão Preto. Ribeirão Preto: FEA, 2001. 19 p. Texto para discussão USP/FEA. (Série Economia 22).

BINI, D. A; DENARDIN, A. A. Correlação e causalidade entre os preços de commodities e energia. In: ENCONTRO DE ECONOMIA DA REGIÃO SUL, 16, 2013, Curitiba. Anais... Curitiba: UFPR. Disponível em: <http://www.anpec.org.br/sul/2013/submissao/files_I/i4-1426be0c0f68f35be990cdd42d5304ca.pdf>. Acesso em: fev. 2015.

BRAGA, Julia de Medeiros. A inflação brasileira na década de 2000 e a importância das políticas não monetárias de controle. Economia e Sociedade, [S.l.], v. 22, n. 3, p. 697-727, dez. 2015. ISSN 1982-3533. Disponível em: <http://periodicos.sbu.unicamp.br/ojs/index.php/ecos/article/ view/8642181>. Acesso em: 16 set. 2016.

BUENO, R. L. S. Econometria de séries temporais. São Paulo: Cengage Learning. 2011.

ENGLE, R. F.; GRANGER, C. W. J. Co-integration and error correction: representation, estimation and testing. Econometrica, Chicago, v. 55, n. 2, p. 251-276, 1987.

ENGLE, R. F.; GRANGER, C. W. J.; HALLMAN, J. J. Merging short-run and long-run forecasts: an application of seasonal cointegration to montly electricity sales forecasting. Journal of Econometrics, New York, v. 40, n. 1, p. 45-62, jan. 1989.

ENDERS, W. Rats handbook for econometric time series. New York: Wiley, 1996. 289 p. 
FERNANDEZ, C. Y. H. Câmbio real e preços de commodities: relação identificada através da mudança de regime cambial. 86 p. Dissertação (Mestrado em Economia) - Departamento de Economia, Pontifícia Universidade Católica, Rio de Janeiro, 2003.

FERRARI FILHO, F.; MODENESI, A. M., Choques de oferta, indexação e política monetária: breves considerações sobre a aceleração inflacionária recente. Economia \& Tecnologia, Curitiba, v. 26, p. 5-13, 2011.

FREITAS, S. M. et al. Análise da dinâmica de transmissão de preços no mercado internacional de farelo de soja, 1990-99. Agricultura em São Paulo, São Paulo, v. 48, n. 1, p. 1-20, 2001.

LORA, E.; POWLL, A.; TAVELLA, P. How will the food price shock affect inflation in Latin America and the Caribbean? Inter-American Development Bank, Department of Research and Chief Economist. Policy brief. Apr. 2011. Disponível em: <http://idbdocs.iadb.org/wsdocs/getdocument. aspx?docnum=36144620 > . Acesso em: 11 nov. 2012.

LUQUE, C. A.; VASCONCELLOS, M. A. S. Manual de economia: equipe de professores da USP. 3. ed. São Paulo: Saraiva, 1998.

MACKINNON, J. G.; HAUG, A. A.; MICHELIS, L. Numerical distribution functions of likelihood ratio tests for cointegration. Journal of Applied Econometrics, 14, p. 563-577, 1999.

MALUF, R. S.; SPERANZA, J. S. Volatilidade dos preços internacionais e inflação de alimentos no Brasil: fatores determinantes e repercussões na segurança alimentar e nutricional. Brasília: Secretaria Nacional de Segurança Alimentar e Nutricional, 2013. 148 p. ISBN: 978-85-60700-61-5.

MARGARIDO, M. A.; BARROS, G. S. C. Transmissão de preços agrícolas internacionais para preços agrícolas domésticos no Brasil. Agricultura em São Paulo, São Paulo, v. 47, n. 2, p. 53-81. 2000.

MARGARIDO, M. A.; SERIGATI, F. C.; PEROSA, B. B. Análise dos mecanismos de transmissão dos preços internacionais de commodities agrícolas sobre o comportamento da taxa de câmbio real no Brasil: uma aplicação de modelos de séries temporais. São Paulo: Escola de Economia de São Paulo, maio 2010. Fundação Getúlio Vargas. Textos para discussão n. 256.

MARQUES, S. A. Índice de preços pagos pela agricultura paulista: construção e cálculo. Agricultura em São Paulo, São Paulo, v. 38, n. 1, p. 1-9, 1991.

MARTINEZ, T. S.; A dinâmica da inflação brasileira: considerações a partir da desagregação do IPCA. Brasília: IPEA, jul. 2011. Comunicados do IPEA, n. 101.

MARTINEZ, T. S.; CERQUEIRA, V. S. Estrutura da inflação brasileira: determinantes e desagregação do IPCA. Rio de Janeiro: IPEA, 2011. Texto para discussão n. 1.634.

MADDALA, G.S. Introduction to econometrics. $2^{\text {nd }}$ ed, Nova Iorque: MacMillan, 1992.

MELO, A. C. Análise do impacto de choques nos preços internacionais das commodities sobre a inflação brasileira. 2010. vii, 52 p. Dissertação (Mestrado em Economia do Setor Público) - Universidade de Brasília, Brasília, 2010.

SAMUELSON, P.; NORDHAUS, W. Macroeconomia. 18. ed. São Paulo: McGraw-Hill, 2005.

SCHWARTSMAN, A. Anatomia de um choque. Valor Econômico, São Paulo, $1^{\circ}$ nov. 2012. Disponível em: <http://www.valor.com.br/opiniao/2888882/anatomia-de-umchoque>. Acesso em: 8 nov. 2012. 
SILVA, F. J. F., FONSECA NETO, F. de A. Inflação pró-pobre no Brasil do real: uma análise regional. Economia Aplicada, Ribeirão Preto, v. 15, n. 1, p. 83-102, 2011. Disponível em: <http:// www.scielo.br/pdf/ecoa/v15n1/a05v15n1.pdf>. Acesso em: 25 nov. 2012.

SOUZA, W. P. S. F.; ANNEGUES, A. C.; MAIA, S. F. Preços de alimentos e dinâmica inflacionária no Brasil: uma splicação do modelo de Vvtores autoregressivos (VAR). Economia e Desenvolvimento, Recife, v. 14, p. 111-125, 2015.

TODA H.; YAMAMOTO, T. Statistical inference in vector autoregressions with possibly integrated processes. Journal of Econometrics, New York, n. 66, p. 225-250, 1995.

\section{Anexo}

Tabela 7 - Resultado do teste de raiz unitária Phillips-Perron para as séries PIB, IPCAF, SELIC, CAMBIO e ICAGR, janeiro de 2003 a dezembro de 2013

\begin{tabular}{l|c|c|c|c|c|c|c|c|c}
\hline \multirow{2}{*}{ Série } & \multicolumn{3}{|c|}{$\begin{array}{c}\text { Modelo com constate } \\
\text { tendência }\end{array}$} & \multicolumn{3}{c|}{ Modelo com constante } & \multicolumn{3}{c}{ Passeio aleatório } \\
\cline { 2 - 10 } & \multicolumn{1}{c|}{$\tau_{\tau}$} & Prob. & lag $^{1}$ & $\tau_{\mu}$ & Prob. & lag $^{1}$ & $\tau$ & Prob. & lag $^{1}$ \\
\hline PIB & $-2,096$ & 0,543 & 0 & $-1,046$ & 0,735 & 4 & 3,281 & 1,000 & 4 \\
IPCAF & $-0,934$ & 0,948 & 0 & 1,930 & 1,000 & 1 & 7,333 & 1,000 & 3 \\
SELIC & $-2,854$ & 0,181 & 3 & $-2,140$ & 0,230 & 3 & $-1,380$ & 0,155 & 5 \\
CAMBIO & $-1,076$ & 0,928 & 7 & $-2,366$ & 0,154 & 6 & $-1,337$ & 0,167 & 6 \\
ICAGR & $-2,622$ & 0,271 & 4 & $-1,307$ & 0,625 & 4 & 0,449 & 0,810 & 6 \\
\hline
\end{tabular}

Fonte: elaborada pelos autores com resultados da pesquisa.

Nota: ${ }^{1}$ Critério para bandwidth (Newey-West automatic) usando Bartlett kernel.

Tabela 8 - Resultado do teste de raiz unitária DF-GLS para as séries PIB, IPCAF, SELIC, CAMBIO e ICAGR, janeiro de 2003 a dezembro de 2013

\begin{tabular}{l|c|c|c|c}
\hline \multirow{2}{*}{ Série } & \multicolumn{2}{|c}{ Modelo com constate e tendência } & \multicolumn{2}{c}{ Passeio aleatório } \\
\cline { 2 - 5 } & $\tau_{\mu}$ & lag $^{1}$ & $\tau$ & lag $^{1}$ \\
\hline PIB & $-2,230$ & 0 & 1,740 & 0 \\
IPCAF & $-0,944$ & 2 & 3,653 & 2 \\
SELIC & $-2,873$ & 3 & $-0,603$ & 3 \\
CAMBIO & $-0,695$ & 0 & $-0,157$ & 0 \\
ICAGR & $-2,288$ & 1 & $-1,688$ & 1 \\
\hline
\end{tabular}

Fonte: elaborada pelos autores com resultados da pesquisa.

Nota: ${ }^{1}$ Lag Length: (Automatic - based on SIC, maxlag=12). 
Tabela 9 - Resultado do teste de raiz unitária NG-Perron para as séries PIB, IPCAF, SELIC, CAMBIO e ICAGR, janeiro de 2003 a dezembro de 2013

\begin{tabular}{|c|c|c|c|c|}
\hline \multirow[t]{2}{*}{ Série } & \multicolumn{2}{|c|}{$\begin{array}{l}\text { Modelo com constate } \\
\text { e tendência }\end{array}$} & \multicolumn{2}{|c|}{$\begin{array}{l}\text { Modelo com } \\
\text { constante }\end{array}$} \\
\hline & $\overline{M Z}_{t}$ & Bandwidth & $\overline{M Z}_{t}$ & Bandwidth \\
\hline \multicolumn{5}{|l|}{ Nível } \\
\hline PIB & $-2,15$ & 0 & 1,92552 & 4 \\
\hline IPCAF & $-0,19$ & 0 & 7,42584 & 1 \\
\hline SELIC & $-2,15$ & 3 & $-0,08084$ & 3 \\
\hline CÂMBIO & $-0,63$ & 7 & $-0,08357$ & 6 \\
\hline ICAGR & $-1,78$ & 4 & $-1,36499$ & 4 \\
\hline \multicolumn{5}{|l|}{ Diferença } \\
\hline PIB & $-5,84556$ *** & 4 & $-6,00649 * * *$ & 3 \\
\hline IPCAF & $-3,0782$ & 26 & $-3,69769 * * *$ & 19 \\
\hline SELIC & $-6,24811^{* * *}$ & 6 & $-5,50774 * * *$ & 6 \\
\hline CÂMBIO & $-3,88332^{* * *}$ & 11 & $-3,65407^{* * *}$ & 7 \\
\hline ICAGR & $-3,97062 * * *$ & 7 & $-3,20999 * * *$ & 7 \\
\hline
\end{tabular}

Fonte: elaborada pelos autores com resultados da pesquisa.

Nota: ${ }^{* *}$ significativo a $1 \%,{ }^{* *}$ significativo a $5 \%$.

Tabela 10 - Resultado do teste de normalidade dos resíduos

\begin{tabular}{l|c|c|c|c|c|c}
\cline { 2 - 6 } & \multicolumn{2}{c|}{ Teste de Jarque-Bera } & \multicolumn{2}{c|}{ Teste de assimetria } & \multicolumn{2}{c}{ Teste de Curtose } \\
\cline { 2 - 6 } & \multicolumn{1}{c|}{$X^{2}$} & Prob. & \multicolumn{1}{c}{$X^{2}$} & Prob. & \multicolumn{1}{c}{$X^{2}$} & Prob. \\
\hline DPIB & 57,077 & 0,000 & 11,173 & 0,001 & 45,904 & 0,000 \\
DIPCAF & 2,660 & 0,264 & 2,452 & 0,117 & 0,208 & 0,648 \\
DSELIC & 0,331 & 0,847 & 0,028 & 0,866 & 0,303 & 0,582 \\
DCAMBIO & 0,196 & 0,907 & 0,013 & 0,910 & 0,183 & 0,669 \\
DICAGR & 10,177 & 0,006 & 1,469 & 0,225 & 8,708 & 0,003 \\
Variáveis conjuntas & 70,442 & 0,000 & 15,136 & 0,010 & 55,305 & 0,000 \\
\hline
\end{tabular}

Fonte: elaborada pelos autores com resultados da pesquisa. 
Tabela 11 - Resultado do teste de LM de autocorrelação

\begin{tabular}{c|c|c|c|c|c}
\hline lag & $X^{2}$ & Prob. & lag & $X^{2}$ & Prob. \\
\hline 1 & 45,188 & 0,008 & 7 & 18,663 & 0,813 \\
2 & 17,865 & 0,848 & 8 & 20,068 & 0,743 \\
3 & 31,536 & 0,172 & 9 & 39,667 & 0,032 \\
4 & 25,109 & 0,456 & 10 & 28,133 & 0,302 \\
5 & 33,165 & 0,127 & 11 & 23,258 & 0,563 \\
6 & 19,864 & 0,754 & 12 & 19,750 & 0,760 \\
\hline
\end{tabular}

Fonte: elaborada pelos autores com resultados da pesquisa. 